\title{
The Skill Research of Interactive Web Site Based on.NET: The Design and Development
}

\author{
Yanwei XU ${ }^{1, a}$, Honghui GONG ${ }^{2}$, Ting ZHANG $^{3}$ \\ 1,2,3 Jiangxi Police College, Nanchang 330200, China \\ axuyanweijx@126.com
}

Keywords: Interactive Web Site; ASP.NET; ADO.NET

\begin{abstract}
With the development of network information technology and multimedia technology, require Web sites with convenient, fast and efficient features, interactive website built into the current research focus. The use of interactive, resource sharing, this paper describes the development of interactive multimedia website based on ADO.NET and ASP.NET technology, the system can update the site content in real time according to need, in order to achieve dynamic data Web server publishing, dynamic, interactive web pages core design and implementation is to build dynamic database, the traditional static Web pages need to manually update the non-online, so traditional page features only stay in the browser and access to knowledge level, and NET technology makes building dynamic database, to complete an interactive web design change too simple.
\end{abstract}

\section{Introduction}

Many sites on the Internet still provides 'static' home page content. Under normal circumstances, the user through the browser to see the page is mostly static. The so-called static refers to the site's home page content is "fixed" in Browser Send to a Web server via HTTP protocol requests, after Web server receives a request, which has been designed static HTML data sent to the browser. a "static" site to update the home page content, you must manually update HTML file data With the development of Web applications, users want to see the request and dynamically generated page, for example in response to a user request to query the database to generate reports [1-2]. According to the conventional method of user request to generate dynamic page has CGI. ISAPI is based browsers and other CGI side response http request activation process, a process corresponding to each request, while at the same time there are a lot of requests, the program diverted system resources, resulting in inefficiencies [3].

Web applications are the most widely used application development technology on the current Internet, which supports real-time information dissemination, flexible security dynamic user interaction and connection with back-end systems. So how to construct a more powerful, applications more flexible, easier to develop Web applications is one of the hotspots of the current Internet development. NET multi-layer architecture is shared by a variety of applications as a set of services, which is a cross-application, reusable platform. On this platform, a variety of applications can be integrated as a component or recombinant to generate new applications. Due to its flexible scalability, ideal for Internet applications will become the mainstream of Web application development platform basis.

\section{Interactive web analytics}

Interactive dynamic site is concerned with respect to static pages. The so-called static pages refers to the script statements contained pages will not run on the server, when visitors to the site through a browser requests the page, the server will not make any changes to it, it sends the page directly to the requesting browser device. In other words, the final content of static pages is determined by the web design and development and fixed down. Upon making web pages, their content will not change. The so-called interactive dynamic web page is dynamically generated HTML code pages. These dynamic content is not HTML code is written in advance by good! But 
when users browse the Web, according to the server-side scripting operation! Then dynamically generate HTML code. In other words, These pages did not exist prior to the user's browser, there is only generated script pages, because the user's request, by a server script temporarily generated according to the different needs of different people server returned to their pages may not unanimous. Interactive the most important feature is its dynamic interactive web pages that page will dynamically responds according to the user's requirements and choices [4-6].

Interactive website refers to site visitors can freely express their views, to work with the site manager or the formation of other visitors interact, communicate, and thus be able to participate in site content and impact of the construction site role played by these acts website. In short, it is the ability to achieve those Internet users to communicate with the network interactive website. The main form of forum BBS, guestbook, site reviews, chat room, blog, instant messaging and so on. Features an interactive website based on site visitors as the center, give full play to the enthusiasm of site visitors, in terms of site construction management, and other practical work under the net with an open and constructive attitude to draw visitors' opinions, promote website Affiliation work and development, through exchange, will view the site manager, position, attitude efficiently transmitted to website visitors.

Interactive website designed to use the main programming language (ASP, PHP, Java, etc.), the majority of use combined with JavaScript scripting language, beautiful pages, Flash can reach into vivid colorful web-based database, page production, plus backstage Control page can generate powerful page, such as online office, online sales, online recruitment, logistics management, personnel management, and other modules.

\section{The interactive web site based on ASP.NET}

ASPNET is a unified Web development platform, to provide developers build enterprise-class Web applications required services. ASPNET largely syntax compatible with ASP, but it also provides a new programming model and structure, is used to generate more secure, scalable, and stable applications. By gradually adding ASP.NET functionality to existing ASP application at any time to enhance the function of the ASP application. ASP.NET is a .NET-based environment, you can use any .NET-compatible language a word (including Visual Basic. NET, C \# and Jscript.NET) to create the application. In addition, any ASP.NET application can use the whole .NET Framework class library. Developers can easily access the advantages of these technologies, including the hosting of the common language runtime environment, type safety, inheritance, and so on. ASP.NET seamlessly work with WYSIWYG (WYSIWYG) HTML editor and other programming tools (including MicrosoftVisualStudio.NET). This not only makes Web development easier, but also to provide all the benefits of these tools have to offer, including the development of server controls can be used to drag and drop GUI Web page and fully integrated debugging support. ASP.NET based entirely on modules and components, has good scalability and customization, data processing is the introduction of many new technologies, it is the significance of these innovative new features, so far beyond the ASP.NET ASP, and also available to Web developers greater flexibility, shorten the development cycle of Web applications.

ASP.NET controls introduced as a declaration based on the model of the server. This is a very different concept, because the control on the server statement, the server can be programmed to be a client-driven events. It sounds incredible, but it is very simple to use. To put an ordinary HTML control into a server control, such as simply "runat = server", as a feature added to the code. Controls run on the server, will allow us to use the ID feature to directly recognize it. This code has a higher readability, because the contents of the form does not have to quote or copy the contents into a variable. While direct reference to the control will be more natural, and thus developed using the page easier. 


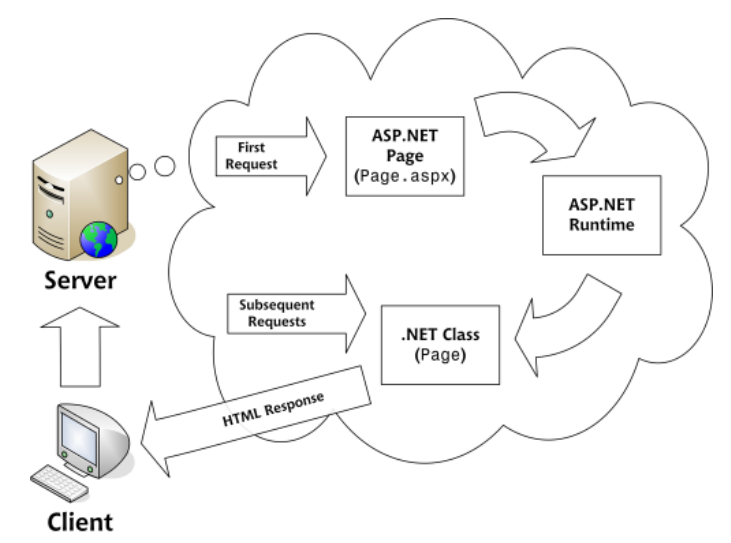

Figure 1. The interactive web site based on ASP.NET

\section{The interactive web site based on ADO.NET}

Use ADO.NET database access the database, you must first contact the database, then issue SQL commands or stored procedure database intend to tell what kind of work, and finally return the required data record from the database. The entire structure shown in Figure 2:

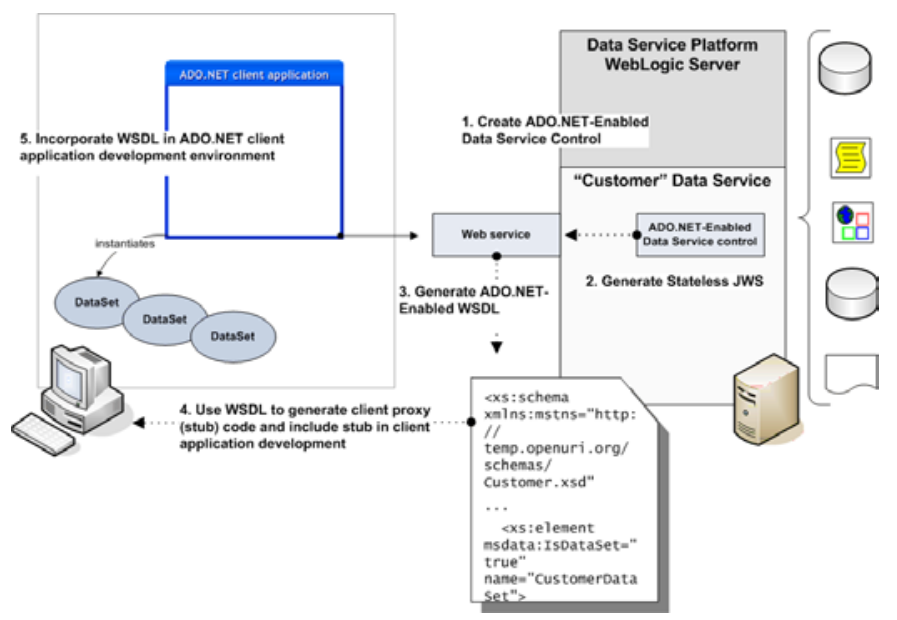

Figure 2. The interactive web site based on ADO.NET

ADO.NET has two core components: Data Set and .NET data provider, each component contains many objects, some of the following major subject discussed. Connection object is mainly used for the connection between open programs and databases. Do not open the database using the connection object, it is unable to obtain data from the database. This object is the lowest level in ADO.NET, you can dynamically generate the object program can also be automatically generated by other objects. Data Set (dataset) is the core of ADO.NET objects, designed to deal with the read-out data from the data store, and offline exist in the local memory. Data Set the same way to manipulate the data obtained from different data sources, regardless of the underlying database is SQL Server or Oracle. In the Data Set can contain any number of Data Table, and a table or view Data Table correspond to each database. Data Table will be responsible for maintaining a data per row, to retain its initial state and the current state, in order to solve the problem of concurrent access to the database. Data Adapter object is used to create, initialize the data table, and Data Set objects combine data stored in memory. Data Adapter objects can be hidden and Connection, Command objects communicate details. Data Adapter Object Data Set lets you save the data to a data source object, you can retrieve data from a data source, you can also save the body to underlying data to add data, delete or modify operation. Data Adapter object contains four different types of Command. Select Command: Data sources used to obtain the records. Insert Command: used to add records to the data source. Update Command: Data sources used to update the records. Delete Command: to remove the data source records. Ben stood when accessing the database through the Connection object completed first connect to the database, and then use the Command object to operate the 
database, and the results are retrieved from the database is placed into the Data Set objects; and finally, the use of an auxiliary control ADO.NET will result.

\section{The application of interactive web site based on .NET}

According to the needs analysis, site design. The fundamental objective of the website design phase is to determine specifically what should achieve the required system, after this stage of the design work, should come to an accurate description of the target system, resulting in the coding phase can be directly used to design a page description language translation into Web applications. In addition, human-computer interface design is an important part of the detailed design. A clear description of the detailed design of software for software developers, directly affect the correctness of their procedures and programming efficiency. Detailed design description for software testing and maintenance personnel are also important documents, so they do not need to read the code, the website will be able to understand the internal program structure.

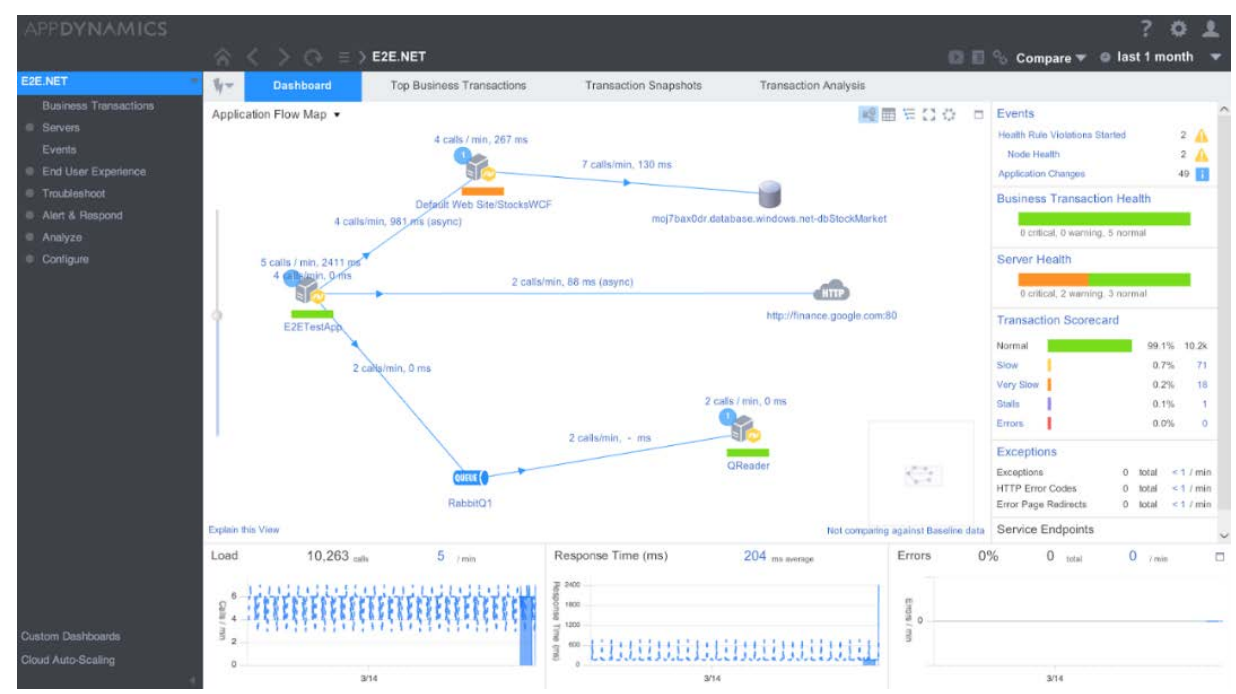

Figure 3 .The case study of interactive web site based on .NET

Figure 3 is based on NET technology design case, the structure of the site is top-down extension, hierarchical modular structure. High-rise module, using transaction-centric design strategy, a large complex system gradually broken down into small relatively simple module; low-level modules, using to transform centric strategy. In addition, in order to improve cohesion modules reduce the coupling between modules, I put the database design as a communication conduit between modules, thereby not only achieve the data sharing and improve the independence of the module, the system can be modified with high sex.

At this stage, to come up with timely prototype model, allowing users to identify and evaluate the prototype, can not meet user requirements for the contents of further modifications and improvements. And repeatedly evaluated the prototype to improve until the system is fully in line with user requirement.

\section{Conclusion}

With the rapid development of the Internet, web has been widely used. Simple static pages has failed to meet the needs of visitors. A professional site not only have a beautiful page, a wealth of information, but also need to have some interactive features visitors Increased content of some viewers personally involved in the site, viewers can mobilize the enthusiasm and initiative, can improve the affinity sites, strengthen exchanges and communication with the user. web page has evolved from static web pages to static pages with interactive Dynamic pages and try, and interactive dynamic web page design and production techniques. This article describes the development of interactive multimedia website ADO.NET and ASP.NET-based technology, the system is able to update the site content in real time according to need, in order to achieve dynamic 
data Web server release, the core dynamic, interactive web design is to build dynamic database and implementation.

\section{Reference}

[1] Sahay R. Hands on with ASP. NET MVC: Covering MVC 6[M]. Vij Books India Pvt Ltd, 2014.

[2] Wang J. Web Database Access Technology Based on ASP. NET[M]//Advances in Multimedia, Software Engineering and Computing Vol. 1. Springer Berlin Heidelberg, 2012: 259-264.

[3] Bao K, Sun Z. The Design of Network Support System for Community Management[J]. International Journal of u-and e-Service, Science and Technology, 2013, 6(2): 53-62.

[4] Ribeiro D J P, Rodrigues J J P C, Han G, et al. SNetGNA communities: A new proposal of web application to online social networking management systems[C]//Communications and Networking in China (CHINACOM), 2014 9th International Conference on. IEEE, 2014: 341-346.

[5] Zhuoyi C, Limin J, Weihua Z. Research and development of the long distance coach management system based on ASP. net technology[C]//Consumer Electronics, Communications and Networks (CECNet), 2012 2nd International Conference on. IEEE, 2012: 1992-1995.

[6] Yang W, Zhang S. A PDM System for Small and Medium Enterprises Based on. Net Framework[C]//Enterprise Systems Conference (ES), 2014. IEEE, 2014: 24-27. 